\title{
Propriedades Psicométricas da Escala de Liderança Servidora
}

\author{
Michelle Morelo Pereira ${ }^{1}$, Maria Cristina Ferreira \\ Universidade Salgado de Oliveira, Niterói-RJ, Brasil
}

\section{RESUMO}

O líder servidor pode ser visto como alguém motivado por um sentimento de servir sua equipe que vai além dos seus próprios interesses. O estudo teve como objetivo adaptar e reunir evidências iniciais de validade de estrutura interna e de relações com outras variáveis da Escala de Liderança Servidora. A amostra foi composta por 432 trabalhadores brasileiros de ambos os sexos (54,6\% do sexo masculino). $\mathrm{Na}$ verificação da estrutura interna, foram testados três modelos, mediante a análise fatorial confirmatória, sendo que o modelo de oito fatores correlacionados foi o que apresentou os melhores índices de ajuste. A maioria das dimensões de liderança servidora apresentou correlações de moderadas a fortes com a liderança autêntica, com o engajamento laboral, com o comprometimento organizacional afetivo e com a satisfação laboral. As evidências iniciais de validade presentemente obtidas recomendam o uso futuro da escala em investigações brasileiras.

Palavras-chave: estilo de liderança; validade estatística; psicometria.

\section{ABSTRACT - Psychometric Properties of the Servant Leadership Scale}

The server leader is seen as someone motivated by a feeling of serving their team that goes beyond their own interests. The aim of the study was to adapt and gather initial evidence of internal structure validity and relationships with other variables for the Servant Leadership Scale. The sample consisted of 432 Brazilian workers of both sexes (54.6\% male). In the verification of the internal structure, three models were tested through confirmatory factor analysis, with the model of eight correlated factors being the one that presented the best fit indices. The majority of the dimensions of servant leadership had moderate to strong correlations with authentic leadership, job engagement, affective organizational commitment, and job satisfaction. The initial evidence of validity obtained strengthens the possibility of the future use of the scale in Brazilian investigations.

Keywords: leadership style; statistical validity; psychometrics.

\section{RESUMEN - Propiedades Psicométricas de La Escala de Liderazgo Servidora}

El líder servidor es visto como alguien motivado por un sentimiento de servir a su equipo que va más allá de sus propios intereses. El estudio tuvo como objetivo adaptar y reunir evidencias iniciales de validez de estructura interna y de relaciones con otras variables de la Escala de Liderazgo Servidor. La muestra fue compuesta por 432 trabajadores brasileños de ambos sexos (54,6\% del sexo masculino). En la verificación de la estructura interna fueron probados tres modelos mediante el análisis factorial confirmatorio, siendo que el modelo de los ocho factores correlacionados fue el que presentó los mejores índices de adaptación. La mayoría de las dimensiones de liderazgo servidor presentó correlaciones de moderadas a fuertes con el liderazgo auténtico, con el compromiso laboral, con el compromiso organizacional afectivo y con la satisfacción laboral. Las evidencias iniciales de validez actualmente obtenidas recomiendan el uso futuro de la escala en investigaciones brasileñas.

Palabras clave: estilo de liderazgo; validez estadística; psicometría.

Nos anos recentes, a corrupção organizacional tem ocupado lugar de destaque nos noticiários brasileiros e internacionais e despertado interesse dos estudiosos das organizações. Acompanhando essa tendência, pesquisas sobre os processos de liderança ética, que tinham diminuído durante a maior parte do século passado, também aumentaram nas últimas décadas (Cuilla, 1998; Downe, Cowell, \& Morgan, 2016).

A liderança ética consiste na demonstração de condutas normativamente adequadas (como ser aberto e honesto), por meio de ações pessoais, e na promoção de tal conduta entre os seguidores (Van Dierendonck, 2011). Ela envolve, portanto, valores como o altruísmo, a compaixão, a honestidade e a justiça, que se encontram inseridos em várias teorias proeminentes na literatura atual sobre liderança, tais como as teorias de liderança autêntica e de liderança servidora (Liden, Wayne, Zhao, \& Henderson, 2008; Walumbwa, Avolio, Gardner, Werning, \& Peterson, 2008). 
De particular interesse para o presente trabalho é a liderança servidora, que consiste em um tipo específico de liderança ética, e tem suas origens na publicação do livro The Servant as Leader, de autoria de Greenleaf (1977). De acordo com o autor, o líder servidor pode ser visto como um servo, isto é, como alguém motivado pelo sentimento de servir a sua equipe que vai além dos seus próprios interesses. Tal definição adiciona à liderança o componente de responsabilidade social e ressalta explicitamente as necessidades dos seguidores (Patterson, 2003).

Em uma análise das diversas dimensões que compõem a liderança servidora, Van Dierendonck (2011) observou a existência de 44 dimensões e procurou sintetizá-las no Modelo de Liderança Servidora, que engloba comportamentos e virtudes já inseridas em outros modelos. O modelo prevê oito características para um líder servidor: empoderamento, humildade, autenticidade, aceitação interpessoal ou perdão, responsabilização, coragem, respaldo e servidão. $\mathrm{O}$ empoderamento diz respeito à promoção de uma atitude proativa e autoconfiante entre os seguidores, que lhes dá a sensação de poder pessoal, valorização e incentivo a seu desenvolvimento pessoal.

A humildade refere-se à colocação do interesse dos seguidores em primeiro lugar, mediante a facilitação de seu desempenho e fornecimento de suporte. O líder servidor admite, portanto, que ele pode se beneficiar da experiência dos outros. A autenticidade, por sua vez, está relacionada à expressão do "verdadeiro eu", de modo consistente com os pensamentos e sentimentos internos, isto é, à fidelidade consigo mesmo, como forma de representar com precisão as próprias intenções (Harter, 2002; Peterson \& Seligman, 2004).

A aceitação interpessoal ou perdão consiste na capacidade de compreender e experimentar os sentimentos dos outros. Ela inclui o elemento de tomada de perspectiva da empatia e caracteriza-se pela adoção cognitiva da perspectiva psicológica de outras pessoas, como, por exemplo, a compaixão e o perdão (George, 2000; Van Dierendonck, 2011).

A responsabilização pode ser definida como a capacidade para responsabilizar as pessoas pelo desempenho, fornecendo aos seguidores limites dentro do quais eles ficam livres para alcançar seus objetivos. Outra característica da liderança servidora é a coragem, que pode ser conceituada como a capacidade de ousar e assumir riscos, ou seja, de experimentar novas abordagens para problemas antigos. No contexto organizacional, a coragem significa ainda desafiar modelos convencionais de comportamentos de trabalho, constituindo-se, portanto, em uma característica que distingue o líder servidor de outros líderes (Greenleaf, 1991; Hernandez, 2008).

O respaldo diz respeito à habilidade do líder de colocar em primeiro lugar o interesse dos outros, dando-lhes o apoio e os créditos necessários. A servidão, por fim, caracteriza-se pela vontade de assumir a responsabilidade pela instituição e de atuar como modelo, mediante o uso de exemplos corretos, que estimulam o interesse dos seguidores de agir. De acordo com Van Dierendonck (2011), a maioria das escalas de liderança servidora incluem apenas aspectos do servir, como ajudar e honrar, e, por esse motivo, não engloba o construto em sua totalidade. Em outras palavras, as escalas destinadas a avaliar a liderança servidora costumam enfatizar sobremaneira a característica de servir, deixando de considerar outras características importantes como, por exemplo, a coragem.

Fundamentando-se em tais considerações, Van Dierendonck e Nuijten (2011) propuseram a Escala de Liderança Servidora, tomando por base o referido Modelo de Liderança Servidora. Em um primeiro estudo com a escala, realizado em uma amostra on-line de 668 holandeses, as análises fatoriais exploratórias resultaram em um instrumento com 28 itens, distribuídos em seis dimensões, que explicaram $65 \%$ da variância.

Em um segundo estudo, foram acrescentados novos itens à escala que passou a ter 39 itens e foi aplicada a 263 funcionários públicos holandeses. $\mathrm{Na}$ análise fatorial confirmatória, um modelo composto pelas oito dimensões preconizadas pela teoria apresentou melhores índices de ajuste que um modelo unifatorial $\left(\chi^{2}(g l)=623,5\right.$ (377); $\mathrm{CFI}=0,93 ; \mathrm{TLI}=0,92 ; \mathrm{SRMR}=0,05 ; \mathrm{RMSEA}=0,05)$, após a exclusão de nove itens. Em outro estudo, realizado com 236 trabalhadores holandeses, a análise fatorial confirmatória apontou que o modelo de oito fatores com um fator geral de segunda ordem $\left(\chi^{2}(g l) 600,1(397)\right.$; $\mathrm{CFI}=0,94 ; \mathrm{TLI}=0,93 ; \mathrm{SRMR}=0,06 ; \mathrm{RMSEA}=0,05)$, distribuídos por 30 itens, apresentou melhores índices de ajuste que os modelos de sete e seis fatores.

Para a validação de construto, a Escala de Liderança Servidora foi aplicada, juntamente com outras medidas de liderança (servidora, transformacional, ética, carismática e troca líder-membro), a uma amostra de 1571 respondentes (1187 holandeses e 384 ingleses). Os resultados obtidos evidenciaram que as dimensões da escala de liderança servidora apresentaram correlações de baixas a moderada com a vitalidade $(0,17$ a 0,33$)$, e de moderadas a fortes, com o engajamento $(0,18$ a 0,43$)$, a satisfação no trabalho $(0,20$ a 0,62$)$ e o comprometimento organizacional $(0,14$ a 0,32$)$.

A Escala de Liderança Servidora foi ainda traduzida e adaptada para a língua espanhola, em uma amostra de 638 trabalhadores de três países (Espanha, Argentina e México) e para a língua portuguesa, em uma amostra de 1107 trabalhadores (Rodríguez-Carvajal, Rivas, Herrero, Moreno-Jiménez, \& Van Dierendonck, 2014; Sousa \& Van Dierendonck, 2014). Os resultados da análise fatorial confirmatória, em ambos os estudos, indicaram que foi corroborada a estrutura de oito fatores de primeira ordem com um fator geral de segunda ordem (liderança servidora).

A consulta às bases de dados nacionais (banco de testes e dissertações CAPES, SciELO e PePSIC), realizada em agosto de 2017, com os descritores scale, servant, 
servant leadership, escala e liderança servidora, indicou apenas dois estudos nacionais sobre a temática. O estudo de Costa (2007) realizou somente a tradução do Servant Leadership Assessment Instrument, de autoria de Dennis e Bocarnea (2005), para o português do Brasil, não tendo investigado suas características psicométricas. Almeida e Faro (2016) adaptaram e reuniram evidências de validade da escala de Liderança Servidora de Barbuto e Wheeler (2006), em amostras brasileiras. No entanto, o estudo referido trabalhou apenas com uma amostra composta por alunos (graduação ou pós-graduação) e servidores efetivos ou terceirizados de uma única instituição de ensino federal.

A realização de estudos capazes de reunir evidências de validade sobre a escala de Liderança Servidora de Van Dierendonck e Nuijten (2011) em uma amostra de trabalhadores em geral, justifica-se, portanto, na medida em que ela ainda não foi objeto de estudos voltados à análise de suas características psicométricas em amostras brasileiras. Acrescente-se a isso o fato de que, dentre os instrumentos disponíveis para a avaliação da liderança, a escala mencionada é a única que inclui todos os elementos essenciais da liderança servidora originalmente propostos por Greenleaf (1996). Igualmente, um estudo dessa natureza poderá incentivar a investigação empírica do construto liderança servidora em amostras brasileiras, ampliando, dessa forma, sua rede nomológica. Fundamentando-se em tais considerações, o presente estudo teve como principal objetivo adaptar e reunir evidências iniciais de validade de estrutura interna, de invariância por sexo e modalidade de coleta de dados e de relações com outras variáveis da Escala de Liderança Servidora, em uma amostra de trabalhadores brasileiros.

$\mathrm{Na}$ análise das correlações com outras variáveis foram utilizadas medidas de avaliação da liderança autêntica, do engajamento no trabalho, do comprometimento organizacional afetivo e da satisfação laboral. Considerando-se que o líder autêntico define-se por atributos de caráter e integridade que conferem direção aos subordinados e os ajudam a encontrar um significado para seus trabalhos e suas vidas (Sobral \& Gimba, 2012), hipotetizou-se que haveria uma correlação positiva de moderada a forte entre a liderança servidora e a liderança autêntica $\left(\mathrm{H}^{1}\right)$.

O engajamento no trabalho diz respeito a um estado psicológico, de natureza afetivo-cognitiva, que se manifesta na dedicação, vigor e absorção ao trabalho (Schaufeli, Salanova, González-Romá, \& Bakker, 2002). O comprometimento organizacional afetivo, por sua vez, corresponde à identificação e envolvimento que o trabalhador manifesta com a organização para a qual trabalha (Van Horn, Taris, Schaufeli, \& Schreurs, 2004). Já a satisfação laboral pode ser definida como um estado positivo que se expressa afetiva e cognitivamente e decorre do trabalho (Van Horn et al., 2004). Partindo-se do pressuposto de que os três construtos referem-se a resultados positivos no trabalho e que o líder servidor pode instigar esses resultados (Van Dierendonck \& Nuijten, 2011), hipotetizou-se que a liderança servidora apresentaria uma correlação positiva de moderada a forte com o engajamento no trabalho $\left(\mathrm{H}^{2}\right)$, o comprometimento organizacional afetivo $\left(\mathrm{H}^{3}\right)$ e a satisfação laboral $\left(\mathrm{H}^{4}\right)$.

\section{Método}

\section{Participantes}

A amostra foi composta por 432 trabalhadores brasileiros de diversas profissões e de ambos os sexos $(54,6 \%$ do sexo masculino), provenientes de todas as regiões do país, com a maioria concentrando-se nos estados do Rio de Janeiro (65,3\%). A idade dos participantes variou de 19 a 68 anos $(M=33,68 ; D P=10,68)$, o tempo de trabalho no emprego atual, de 1 a 36 anos $(M=6,50 ; D P=6,01)$, e o tempo de trabalho total, de 1 a 45 anos $(M=12,19$; $D P=9,51)$. A faixa salarial da maioria variou de 1 a 3 salários mínimos $(41,0 \%)$ e de 3 a 5 salários mínimos $(25,3 \%)$. Os participantes trabalhavam, em sua maioria, no setor público $(51,5 \%)$, em cargos de nível administrativo ou operacional (52,6\%). No que diz respeito à escolaridade, $62 \%$ possuíam ensino superior completo, sendo que $37,9 \%$ tinham também pós-graduação. Quanto ao estado civil, a maioria era casada $(46,8 \%)$ e não possuía filhos (65,3\%). Para inclusão no estudo, utilizou-se o critério de o participante estar trabalhando e possuir pelo menos um ano de experiência em sua respectiva função.

\section{Instrumentos}

A liderança servidora foi medida pela Escala de Liderança Servidora (SLS) de Van Dierendonck e Nuijten (2011), composta por 30 itens, distribuídos em oito dimensões, a serem respondidos em uma escala tipo Likert de seis pontos, variando de 1 (Nunca) a 6 (Sempre). Exemplo de item: "Meu chefe imediato aprende a partir das críticas". Pelo fato dessa escala não possuir uma versão para o contexto brasileiro, foi necessária a sua tradução do inglês para o português, por dois especialistas bilíngues. As versões em português foram compiladas e em seguida, foi realizado o backtranslation, ou seja, a tradução reversa do português para o inglês e, em seguida, a versão traduzida foi comparada com a original por um expert. Tal versão preliminar foi aplicada a um grupo focal de estudantes de psicologia, com o objetivo de verificar se as instruções eram claras e se os termos presentes nos itens estavam adequados (Borsa, Damásio, \& Bandeira, 2012). Na versão final, foram feitos alguns ajustes como a inversão do item 13, para maior compreensão de seu conteúdo.

A liderança autêntica foi avaliada pela Escala de Liderança Autêntica (ALQ) de Walumbwa et al. (2008), adaptada para amostras brasileiras por Cervo, Mónico, Santos, Hutz, e Pais (2016). O instrumento compõe-se de 16 itens, distribuídos em quatro dimensões: 
autoconsciência, transparência, moral e ética e processamento balanceado. Tais itens são respondidos em escalas Likert, variando de nunca a frequentemente. Exemplo de item: "Meu chefe imediato admite erros quando eles ocorrem". No estudo original de adaptação, a escala apresentou índices de consistência interna iguais a: 0,73 (autoconsciência); 0,77 (transparência relacional); 0,73 (moral e ética); 0,70 (processamento balanceado). No estudo atual, eles foram respectivamente iguais a 0,$84 ; 0,88 ; 0,88 ; 0,87$.

O engajamento no trabalho foi avaliado pela versão reduzida da Escala de Engajamento no Trabalho (UWES), de Schaufeli e Bakker (2003), adaptada para o contexto brasileiro por Ferreira et al. (2016). Ela é composta de nove itens (exemplo de item: "Estou entusiasmado com meu trabalho"), a serem respondidos em escalas tipo Likert de sete pontos, variando de nunca (0) a diariamente (6). A consistência interna da escala, no estudo de Ferreira et al. (2016), avaliada pelo alfa de Cronbach, foi igual a 0,91 . No presente estudo, ele foi de 0,95.

O comprometimento organizacional afetivo no trabalho foi avaliado pela Escala de Comprometimento Afetivo de Meyer e Allen (1991), validada em amostras brasileiras por Ferreira et al. (2002). A escala é unifatorial, sendo composta por seis itens, a serem respondidos em escalas de cinco pontos, variando de discordo totalmente (1) a concordo totalmente (5). Exemplo de item: "Sinto-me, de fato, pertencendo à minha organização". No processo de validação original em amostras brasileiras, a escala obteve um índice de consistência interna igual a 0,85 . No atual estudo, ele foi igual a 0,90 .

A satisfação no trabalho foi medida pela Escala de Satisfação Geral no Trabalho de Silva e Ferreira (2009). O instrumento é unifatorial e composto por cinco itens, a serem respondidos em escalas Likert, variando de discordo fortemente (1) a concordo fortemente (5). Exemplo de item: "Sinto-me satisfeito com meu trabalho atual". No estudo de validação original, o referido instrumento obteve índice de consistência interna de 0,80 . No presente estudo, tal índice foi de 0,92 .

\section{Procedimentos}

A pesquisa foi submetida ao Comitê de Ética em Pesquisa da instituição dos autores e aprovada com o $\mathrm{n}^{\mathrm{o}}$ 2.217.569. A coleta de dados foi realizada por meio de duas versões: lápis e papel $(25,46 \%)$ e versão on-line (74,54\%). Na versão lápis e papel, os questionários foram aplicados em uma instituição federal, entregues e recolhidos logo após o seu preenchimento. A versão on-line foi elaborada no aplicativo Google Drive, com uma versão idêntica à impressa e os convites à participação foram enviados por e-mail ou postados no Facebook. Em ambas as situações houve concordância prévia sobre a participação na pesquisa, mediante o preenchimento do Termo de Consentimento Livre e Esclarecido (TCLE).

\section{Análise de dados}

Com o intuito de verificar a estrutura interna da Escala de Liderança Servidora, procedeu-se à análise fatorial confirmatória, por meio da Modelagem de Equações Estruturais, utilizando-se o software R, o pacote lavaan (Rosseel, 2012) e o estimador Weighted Least Square Mean and Variance Adjusted (WLSMV). Cumpre enfatizar que a escolha de tal estimador deveu-se ao fato de ele ser considerado um método de extração robusto para dados ordinais e de não fazer exigências quanto à distribuição normal das variáveis observadas (Muthén \& Muthén, 2010), uma vez que o teste de Shapiro-Wilk indicou a não normalidade da distribuição multivariada dos dados da amostra. Para a avaliação do ajuste do modelo aos dados, foram considerados os seguintes indicadores: Root-MeanSquare Error of Approximation (RMSEA), Standardized Root Mean Square Residual (SRMR), Comparative Fit Index (CFI) e Tucker-Lewis Index (TLI) (Byrne, 2001). Foram adotados, como bons índices de ajustes, valores abaixo de 0,06 para o RMSEA e SRMR e valores maiores que 0,95 para o CFI e para o TLI (Hu \& Bentler, 1999). A consistência interna do instrumento foi analisada por meio do coeficiente alfa de Cronbach. Geralmente, considera-se que o intervalo ideal de valores alfa esteja entre $0,80 \mathrm{e}$ 0,90 (Streiner, 2003). A probabilidade de erro foi estabelecida em $5 \%$.

A invariância referente ao sexo e aos dois diferentes métodos de coleta de dados (lápis e papel e questionário on-line) foi testada por meio da análise de invariância multigrupo, com auxilio do software R e do pacote semTools (Jorgensen, Pornprasertmanit, Schoemann, \& Rosseel, 2018). Foram avaliadas a invariância configural $\left(\mathrm{M}^{1}\right)$, métrica $\left(\mathrm{M}^{2}\right)$, escalar $\left(\mathrm{M}^{3}\right)$ e residual $\left(\mathrm{M}^{4}\right)$. Para tanto, foram analisados os aumentos no $\Delta \mathrm{CFI}$, com valores inferiores a 0,01 sendo considerados como invariantes (Damásio, 2013).

A relação da escala com outras variáveis foi investigada por meio de correlações ( $\rho$ de Spearman) entre os escores do instrumento e os escores globais das medidas a ele relacionadas. Optou-se pelo uso de tal coeficiente correlação pelo fato de ele não fazer nenhum pressuposto sobre a distribuição normal da amostra e considera que os dados devem ser pelo menos ordinais (Chen \& Popovich, 2002).

\section{Resultados}

Para a verificação da validade de estrutura interna da escala, foram testados três modelos: oito fatores correlacionados $\left(\chi^{2}(g l)=534,717(377)\right.$; TLI $=0,90 ; \mathrm{CFI}=0,90$; RMSEA $=0,06 ;$ SRMR $=0,05)$, conforme o modelo de oito fatores de primeira ordem com um fator geral (liderança servidora) de segunda ordem $\left(\chi^{2}(g l)=686,941(397)\right.$; $\mathrm{TLI}=0,88 ; \mathrm{CFI}=0,86 ; \mathrm{RMSEA}=0,07 ; \mathrm{SRMR}=0,06)$; modelo unifatorial $\left(\chi^{2}(g l)=731,347\right.$ (350); TLI $=0,82$; $\mathrm{CFI}=0,83 ; \quad \mathrm{RMSEA}=0,08 ; \quad \mathrm{SRMR}=0,07)$. 
Todos os modelos apresentaram bons índices de ajuste, com pequenas diferenças em termos estatísticos. Sendo assim, optou-se pela adoção do modelo de oito fatores correlacionados, em virtude de ele apresentar melhores índices de ajuste, em comparação com os demais e de implicar a retirada de um menor número de itens e de maior correspondência com o modelo teórico de Van Dierendonck (2011).

$\mathrm{Na}$ Tabela 1, são apresentadas as cargas fatoriais não padronizadas e os intervalos de confiança dos itens, no modelo de oito fatores correlacionados. A inspeção dessa tabela revela que, em um intervalo de confiança de 95\%, não há nenhum valor zero, bem como todos os valores referentes à razão crítica são superiores a 1,96, em uma indicação de que todos os parâmetros avaliados são significativamente diferentes de zero. Acrescente-se a isso o fato de que todas as cargas fatoriais padronizadas (Tabela 1) foram superiores a 0,50 (Hair, Hult, Ringle, \& Sarstedt, 2016), à exceção dos itens $7(0,24)$ e $13(0,31)$. Nesse sentido, esses itens foram retirados do modelo, na medida em que eles não resultaram em perda de precisão das dimensões. $\mathrm{O}$ modelo final ajustado apresentou, portanto, 28 itens, distribuídos em oito fatores correlacionados, com os seguintes índices de ajuste: $\chi^{2}(g l)=247$, 266 (322); TLI=0,94; $\quad$ CFI $=0,95 ; \quad$ RMSEA $=0,05$; $\mathrm{SRMR}=0,04$.

Tabela 1

Cargas Fatoriais dos Itens da Escala de Liderança Servidora

\begin{tabular}{|c|c|c|c|c|c|}
\hline & $\begin{array}{c}\text { Carga fatorial } \\
\text { não padronizada }\end{array}$ & Erro padrão & Razão crítica & $\begin{array}{c}\text { Intervalo de } \\
\text { confiança }(95 \%)\end{array}$ & $\begin{array}{l}\text { Carga fatorial } \\
\text { padronizada }\end{array}$ \\
\hline \multicolumn{6}{|c|}{ Empoderamento } \\
\hline Item 1 & - & - & - & - & 0,76 \\
\hline Item 2 & 1,207 & 0,06 & 20,80 & $1,09-1,32$ & 0,78 \\
\hline Item 3 & 1,204 & 0,05 & 22,93 & $1,10-1,31$ & 0,89 \\
\hline Item 4 & 1,249 & 0,05 & 23,09 & $1,14-1,35$ & 0,89 \\
\hline Item 12 & 1,092 & 0,05 & 21,47 & $0,99-1,19$ & 0,77 \\
\hline Item 20 & 1,053 & 0,06 & 18,33 & $0,94-1,16$ & 0,77 \\
\hline Item 27 & 1,148 & 0,05 & 21,21 & $1,04-1,25$ & 0,84 \\
\hline \multicolumn{6}{|l|}{ Respaldo } \\
\hline Item 5 & - & - & - & - & 0,76 \\
\hline Item 13 & 0,439 & 0,08 & 5,72 & $0,20-0,59$ & 0,31 \\
\hline Item 21 & 0,684 & 0,06 & 10,72 & $0,56-0,81$ & 0,51 \\
\hline \multicolumn{6}{|c|}{ Responsabilização } \\
\hline Item 6 & - & - & - & - & 0,73 \\
\hline Item 14 & 1,104 & 0,07 & 15,44 & $0,96-1,25$ & 0,80 \\
\hline Item 22 & 0,947 & 0,07 & 13,35 & $0,81-1,09$ & 0,72 \\
\hline \multicolumn{6}{|l|}{ Perdão } \\
\hline Item 7 & - & - & - & - & 0,24 \\
\hline Item 15 & 3,273 & 1,17 & 2,26 & $0,98-5,57$ & 0,79 \\
\hline Item 23 & 2,807 & 1,16 & 2,41 & $0,53-5,09$ & 0,73 \\
\hline \multicolumn{6}{|l|}{ Coragem } \\
\hline Item 8 & - & - & - & - & 0,81 \\
\hline Item 16 & 0,99 & 0,06 & 15,75 & $0,87-1,11$ & 0,77 \\
\hline \multicolumn{6}{|c|}{ Autenticidade } \\
\hline Item 9 & - & - & - & - & 0,79 \\
\hline Item 17 & 1,115 & 0,05 & 21,80 & $1,02-1,21$ & 0,83 \\
\hline Item 24 & 0,841 & 0,05 & 16,47 & $0,74-0,94$ & 0,67 \\
\hline Item 28 & 1,119 & 0,05 & 21,18 & $1,02-1,22$ & 0,83 \\
\hline \multicolumn{6}{|c|}{ Humildade } \\
\hline Item 10 & - & - & - & - & 0,87 \\
\hline Item 18 & 0,998 & 0,04 & 28,13 & $0,93-1,07$ & 0,86 \\
\hline Item 25 & 1,032 & 0,03 & 30,17 & $0,97-1,10$ & 0,85 \\
\hline Item 29 & 1,071 & 0,04 & 29,79 & $1,00-1,14$ & 0,91 \\
\hline Item 30 & 1,073 & 0,03 & 32,89 & $1,00-1,14$ & 0,90 \\
\hline
\end{tabular}


Tabela 1 (continuação)

Cargas Fatoriais dos Itens da Escala de Liderança Servidora

\begin{tabular}{lccccc}
\hline & $\begin{array}{c}\text { Carga fatorial } \\
\text { não padronizada }\end{array}$ & Erro padrão & Razão crítica & $\begin{array}{c}\text { Intervalo de } \\
\text { confiança (95\%) }\end{array}$ & $\begin{array}{c}\text { Carga fatorial } \\
\text { padronizada }\end{array}$ \\
\hline Servidão & & & & - & 0,86 \\
Item 11 & - & - & - & $0,79-0,95$ & 0,75 \\
Item 19 & 0,871 & 0,04 & 22,42 & $0,89-1,04$ & 0,79 \\
Item 26 & 0,964 & 0,04 & 25,62 & 04 \\
\hline
\end{tabular}

Nota. Optou-se pela exclusão dos itens 7 e 13, por apresentaram cargas fatoriais abaixo de 0,50

$\mathrm{Na}$ análise de invariância multigrupo por sexo, os resultados indicaram que, para os quatro modelos testados (invariância configural $\left(\mathrm{M}^{1}\right)=0,905$; métrica $\left(\mathrm{M}^{2}\right)=0,905$; escalar $\left(\mathrm{M}^{3}\right)=0,903$; residual $\left.\left(\mathrm{M}^{4}\right)=0,898\right)$ não houve variações do CFI superiores a 0,01 . Resultados semelhantes foram encontrados para a análise de invariância por tipo de coleta de dados (on-line e lápis e papel) $\left(\mathrm{M}^{1}=0,884 ; \mathrm{M}^{2}=0,883 ; \mathrm{M}^{3}=0,874 ; \mathrm{M}^{4}=0,864\right)$, em que, não houve, também, variações do CFI superiores a 0,01 . Os índices de consistência interna das oito dimensões foram iguais a: empoderamento $-\alpha=0,93$; humildade $\alpha=0,94$; respaldo $-\alpha=0,56$; servidão $-\alpha=0,84$; responsabilização $-\alpha=0,80$; autenticidade $-\alpha=0,86$; perdão $\alpha=0,62$; coragem $-\alpha=0,77$.

Para a verificação das correlações da escala de Liderança Servidora com outras variáveis, calcularam-se inicialmente as médias aritméticas simples, os desvios padrão e as correlações entre as diferentes escalas do estudo (Tabela 2). Conforme previsto na hipótese 1, todas as dimensões da liderança servidora apresentaram correlações moderadas a fortes com as quatro dimensões da liderança autêntica. A dimensão perdão, porém, apresentou correlações fracas com as dimensões da liderança autêntica $(0,12$ a 0,27$)$. Com relação à hipótese 2 , as dimensões de respaldo e perdão da liderança servidora apresentaram correlações fracas com o engajamento laboral $(\rho=0,24$; $p<0,01 ; \rho=0,11 ; p<0,05$, respectivamente), enquanto as demais dimensões da liderança servidora apresentaram correlações de moderadas a fortes $(0,32$ a 0,41$)$ com o engajamento laboral, conforme previsto.

Resultados semelhantes foram encontrados nas hipóteses 3 e 4, visto que as dimensões empoderamento, humildade, servidão, responsabilização e autenticidade da liderança servidora apresentaram correlações positivas de moderadas a fortes com o comprometimento organizacional positivo e a satisfação no trabalho, respectivamente, enquanto as dimensões de respaldo, perdão e coragem apresentaram correlações fracas com o comprometimento organizacional afetivo e com a satisfação no trabalho (ver Tabela 2).

Tabela 2

Correlações entre as Variáveis do Estudo

\begin{tabular}{|c|c|c|c|c|c|c|c|c|}
\hline & Média & $D P$ & 1 & 2 & 3 & 4 & 5 & 6 \\
\hline 1. Satisfação Laboral & 3,13 & 1,21 & - & & & & & \\
\hline 2. Comprometimento & 3,15 & 1,13 & $0,67^{* *}$ & - & & & & \\
\hline 3. Engajamento & 3,70 & 1,48 & $0,73^{* *}$ & $0,67^{* *}$ & & & & \\
\hline 4. Autoconsciência & 3,13 & 1,11 & $0,30^{* *}$ & $0,30^{* *}$ & $0,75^{* *}$ & - & & \\
\hline 5. Transparência & 3,24 & 1,08 & $0,29 * *$ & $0,35^{* *}$ & $0,38^{* *}$ & $0,77^{* *}$ & - & \\
\hline 6. Moral Ética & 3,04 & 1,20 & $0,28^{* *}$ & $0,27^{* *}$ & $0,36^{* *}$ & $0,78^{* *}$ & $0,73^{* *}$ & - \\
\hline 7. Processamento & 2,91 & 1,18 & $0,36^{* *}$ & $0,32^{* *}$ & $0,39^{* *}$ & $0,78^{* *}$ & $0,75^{* *}$ & $0,83^{* *}$ \\
\hline 8. Empoderamento & 3,74 & 1,49 & $0,37^{* *}$ & $0,41^{* *}$ & $0,46^{* *}$ & $0,75^{* *}$ & $0,74^{* *}$ & $0,74^{* *}$ \\
\hline 9. Humildade & 3,37 & 1,46 & $0,31^{* *}$ & $0,33^{* *}$ & $0,39^{* *}$ & $0,80^{* *}$ & $0,74^{* *}$ & $0,82^{* *}$ \\
\hline 10. Respaldo & 3,10 & 1,34 & $0,22^{* *}$ & $0,20^{* *}$ & $0,24^{* *}$ & $0,55^{* *}$ & $0,53^{* *}$ & $0,56^{* *}$ \\
\hline 11. Servidão & 3,73 & 1,50 & $0,34^{* *}$ & $0,37^{* *}$ & $0,43^{* *}$ & $0,76^{* *}$ & $0,77^{* *}$ & $0,81^{* *}$ \\
\hline 12. Responsabilização & 3,95 & 1,35 & $0,30^{* *}$ & $0,32^{* *}$ & $0,35^{* *}$ & $0,59^{* *}$ & $0,60^{* *}$ & $0,58^{* *}$ \\
\hline 13. Autenticidade & 3,44 & 1,39 & $0,34^{* *}$ & $0,37^{* *}$ & $0,40^{* *}$ & $0,76^{* *}$ & $0,78^{* *}$ & $0,77^{* *}$ \\
\hline 14. Perdão & 3,56 & 1,39 & 0,06 & $0,11^{*}$ & $0,11^{*}$ & $0,12^{* *}$ & $0,27^{* *}$ & $0,13^{* *}$ \\
\hline 15. Coragem & 3,57 & 1,50 & $0,27^{* *}$ & $0,27^{* *}$ & $0,32^{* *}$ & $0,55^{* *}$ & $0,64^{* *}$ & $0,58^{* *}$ \\
\hline
\end{tabular}


Tabela 2 (continuação)

Correlações entre as Variáveis do Estudo

\begin{tabular}{|c|c|c|c|c|c|c|c|c|}
\hline & 7 & 8 & 9 & 10 & 11 & 12 & 13 & 14 \\
\hline \multicolumn{9}{|l|}{ 1. Satisfação Laboral } \\
\hline \multicolumn{9}{|l|}{ 2. Comprometimento } \\
\hline \multicolumn{9}{|l|}{ 3. Engajamento } \\
\hline \multicolumn{9}{|l|}{ 4. Autoconsciência } \\
\hline \multicolumn{9}{|l|}{ 5. Transparência } \\
\hline \multicolumn{9}{|l|}{ 6. Moral Ética } \\
\hline 7. Processamento & - & & & & & & & \\
\hline 8. Empoderamento & $0,74^{* *}$ & - & & & & & & \\
\hline 9. Humildade & $0,81^{* *}$ & $0,86^{* *}$ & - & & & & & \\
\hline 10. Respaldo & $0,54^{* *}$ & $0,64^{* *}$ & $0,66^{* *}$ & - & & & & \\
\hline 11. Servidão & $0,80^{* *}$ & $0,85^{* *}$ & $0,84^{* *}$ & $0,59^{* *}$ & - & & & \\
\hline 12. Responsabilização & $0,59^{* *}$ & $0,79^{* *}$ & $0,68^{* *}$ & $0,58^{* *}$ & $0,70^{* *}$ & - & & \\
\hline 13. Autenticidade & $0,78^{* *}$ & $0,83^{* *}$ & $0,85^{* *}$ & $0,62^{* *}$ & $0,83^{* *}$ & $0,69^{* *}$ & - & \\
\hline 14. Perdão & $0,13^{* *}$ & $0,19^{* *}$ & $0,13^{* *}$ & $0,23^{* *}$ & $0,22^{* *}$ & $0,33^{* *}$ & $0,22^{* *}$ & - \\
\hline 15. Coragem & $0,61^{* *}$ & $0,66^{* *}$ & $0,59^{* *}$ & $0,46^{* *}$ & $0,66^{* *}$ & $0,58^{* *}$ & $0,65^{* *}$ & $0,35^{* *}$ \\
\hline
\end{tabular}

\section{Discussão}

O presente trabalho teve como objetivo adaptar e reunir evidências iniciais de validade de estrutura interna, de invariância por sexo e modalidade de coleta de dados e de relações com outras variáveis da Escala de Liderança Servidora, em uma amostra de trabalhadores brasileiros. Após a análise fatorial confirmatória, observou-se que o modelo de melhor ajuste foi o de oito dimensões correlacionadas e ajustado. Apesar de tal escala apresentar variações em sua estrutura para diferentes amostras (Sousa \& Van Dierendonk, 2015), os achados deste estudo confirmam o modelo teórico da Liderança Servidora de Van Dierendonck e Nuijten (2011), no que diz respeito ao fato de a escala possuir oito dimensões, embora dois itens tenham sido excluídos.

A escala também apresentou invariância por sexo e por tipo de coleta (on-line ou lápis e papel), em uma demonstração de que há equivalência de respostas entre homens e mulheres, e entre os questionários aplicados presencialmente ou por meio virtual. Tais resultados evidenciam, portanto, que as diferenças médias do teste não estão enviesadas (Damásio, 2013).

Os índices de consistência interna para a maioria das dimensões (empoderamento, humildade, servidão, responsabilização, autenticidade e coragem) foram considerados de bons a ótimos. Entretanto, as dimensões de respaldo e de perdão apresentaram consistência interna inferior a desejável, mesmo com o ajuste e exclusão dos itens com baixas cargas fatoriais, além de que seus respectivos itens mostraram-se pouco correlacionados, em mais uma indicação de consistência interna fraca. Os resultados encontrados para essas dimensões refletem o fato de que elas provavelmente sofreram maior influência da variância de erros aleatórios
(Hayes, 1998), razão pela qual estariam apresentando índices de consistência interna mais baixos, existindo assim, elevada probabilidade de que as medidas para essas dimensões não sejam confiáveis e/ou possuam erro de medida elevado, aumentando as chances de resultados não significativos (Marôco \& Garcia- Marques, 2006).

$\mathrm{Na}$ avaliação das relações da escala com outros construtos a ela correlatos, a hipótese 1 previa a existência de uma correlação forte entre a liderança servidora e a liderança autêntica. Quase todas as dimensões da escala de Liderança Servidora apresentaram correlações significativas e fortes com as quatro dimensões da escala de Liderança Autêntica, em uma indicação de que, quanto maiores os índices de liderança autêntica, maiores serão também os índices de liderança servidora. A única exceção deveu-se à dimensão perdão, que apresentou correlações fracas com todas as quatro dimensões da escala de Liderança Autêntica.

Tais resultados vão ao encontro de estudos anteriores, no qual foram obtidas relações positivas fortes entre as dimensões da escala de Liderança Servidora e uma medida de liderança ética, à exceção da dimensão perdão, com a qual foi observada apenas uma correlação moderada entre essas escalas (Van Dierendonck \& Nuijten, 2011). Considerando-se que a liderança autêntica pode ser vista como uma das vertentes da liderança ética, seria de se esperar que ela também apresentasse correlações fortes com a liderança servidora, na medida em que ambas compartilham algumas características, tais como cuidados com os funcionários e integridade. Cumpre ressaltar, porém, que a liderança autêntica é mais diretiva na orientação do líder para com seus subordinados, enquanto a liderança servidora focaliza-se, sobretudo, no desenvolvimento dos seguidores (Sobral \& Gimba, 2012). 
As hipóteses 2, 3 e 4 previam que a liderança servidora apresentaria correlações positivas de moderadas a fortes com o engajamento, o comprometimento organizacional afetivo e a satisfação laboral, respectivamente. Essas hipóteses também foram parcialmente confirmadas, visto que as dimensões de empoderamento, humildade, servidão, responsabilização e autenticidade apresentaram correlações significativas de moderadas a fortes com todos esses resultados positivos em relação ao trabalho.

Esses achados vão ao encontro do estudo de Van Dierendonck e Nuijten (2011), que também observaram correlações de moderadas a fortes entre a liderança servidora, o engajamento, o comprometimento organizacional afetivo e a satisfação laboral. Outros estudos também têm reunido evidências de que estilos de liderança opostos à liderança servidora e caracterizados por líderes controladores, menos favoráveis e com pouca habilidade de dar feedback, estão relacionados a níveis mais baixos de bem-estar (Van Dierendonck, Haynes, Borrill, \& Stride, 2004). Tais resultados indicam, portanto, que o comportamento dos líderes servidores é benéfico para o engajamento, para a satisfação no trabalho e para o comprometimento de seus subordinados. Em outras palavras, os líderes servidores tendem a colaborar para a obtenção de maiores níveis de resultados positivos de seus subordinados, em função de seu comportamento desempenhar papel central no ambiente social das organizações (Van Dierendonck \& Nuijten, 2011).

Resta, porém, discutir o fato de as dimensões de coragem, respaldo e perdão não terem apresentado correlações moderadas a fortes com resultados positivos do trabalho, conforme previsto, tendo, ao contrário, apresentado correlações de fracas a moderadas. Resultados semelhantes foram observados no estudo de Sousa e Van Dierendonck (2014), no qual as dimensões de perdão e coragem também apresentaram correlações fracas com a liderança servidora. Um motivo para tal achado pode estar relacionado à natureza dessas dimensões, ou seja, ao fato de os sinais de coragem, perdão e respaldo não serem provavelmente tão visíveis em uma rotina de trabalho diário e emergirem apenas em certas circunstâncias especiais e de forma não consistente para todos os trabalhadores, o que dificultaria sua relação sistemática com resultados positivos do trabalho (Sousa \& Van Dierendonck, 2014).

Uma das limitações encontradas neste estudo foi a baixa consistência interna de duas dimensões da escala (respaldo e perdão), bem como o menor impacto das dimensões de perdão, respaldo e coragem no modelo final. Sugere-se, portanto, novos estudos para o refinamento da escala, com a adição de novos itens nessas dimensões ou a retirada delas. Considerando-se, outrossim, que a atual pesquisa incluiu categorias ocupacionais diversificadas, seria interessante que estudos futuros reunissem evidências adicionais sobre a invariância da escala, mediante a comparação de sua estrutura interna em grupos ocupacionais específicos.

Estudos futuros poderiam ainda aprofundar a rede nomológica do construto da liderança servidora, especialmente no que diz respeito a seu impacto sobre o bem-estar no trabalho. De todo modo, as evidências iniciais de validade presentemente obtidas recomendam o uso futuro da Escala de Liderança Servidora em investigações brasileiras destinadas a avaliar o grau de liderança servidora e suas implicações para o bem-estar dos trabalhadores.

\section{Referências}

Almeida, S. P., \& Faro, A. (2016). Tradução, adaptação e validação do Servant Leadership Questionnaire (Escala de Liderança Servidora). Revista Psicologia: Organizações e Trabalho, 16(3), 285-297. doi: 10.17652/rpot/2016.3.11929

Barbuto, J. E., Jr., \& Wheeler, D. W. (2006). Scale development and construct clarification of servant leadership. Group and Organizational Management, 31(3), 300-326. doi: 10.1177/1059601106287091

Borsa, J. C., Damásio, B. F., \& Bandeira, D. R. (2012). Adaptação e validação de instrumentos psicológicos entre culturas: Algumas considerações. Paidéia, 22(53), 423-432. doi: 10.1590/S0103-863X2012000300014

Byrne, B. M. (2001). Structural equation modeling with AMOS: Basic concepts, applications and programming. Mahwah, NJ: Erlbaum.

Cervo, C. S., Mónico, L. S. M., Santos, N. R., Hutz, C. S., \& Pais, L. (2016). Authentic Leadership Questionnaire: Invariance between samples of Brazilian and Portuguese employees. Psicologia: Reflexão e Crítica, 29(1), 29-40. doi: 10.1186/s41155-016-0046-4

Chen, P. Y., \& Popovich, P. M. (2002). Correlation: Parametric and nonparametric measures. Thousand Oaks, CA: Sage Publications

Costa, M. G. D. (2007). Em busca de um modelo brasileiro de mentoria e liderança: O caso de uma organização de Call Center (Dissertação de Mestrado). Faculdade Boa Viagem, Recife-PE. Recuperado de http//favip.edu.br/arquivos/462012171215.pdf

Cuilla, J. B. (1998). Leadership ethics: Mapping the territory. In J. B. Cuilla (Ed.), Ethics, the teart of leadership (pp. 3-25). Westport, CT: Quorum Books.

Damásio, B. F. (2013). Contribuições da análise fatorial confirmatória multigrupo (AFCMG) na avaliação de invariância de instrumentos psicométricos. Psico-USF, 18(2), 211-220. doi: 10.1590/S1413-82712013000200005

Dennis, R. S., \& Bocarnea, M. (2005). Development of the servant leadership assessment instrument. Leadership and Organization Development Journal, 26(8), 600-615. doi: 10.1108/01437730510633692.

Downe, J. Cowell, R., \& Morgan, K. (2016). What determines ethical behavior in public organizations: Is it rules or leadership? Public Administration Review, 76(6), 898-909. doi: 10.1111/puar.12562

Ferreira, M. C., Valentini, F., Damásio, B. F., Mourão, L., Porto, J., Chinelato, R. S. C., Novaes, V. P., \& Pereira, M. M. (2016). Evidências adicionais de validade da UWES-9 em amostras brasileiras. Estudos de Psicologia, 21(4), 435-445. doi: 10.5935/1678-4669.20160042 
George, J. M. (2000). Emotions and leadership: The role of emotional intelligence. Human Relations, 53(8), 1027-1055. doi: 10.1177/0018726700538001

Greenleaf, R. K. (1977). Servant leadership: A journey into the nature of legitimate power and greatness. New York: Paulist Press.

Greenleaf, R. K. (1991). The servant as leader. Indianapolis, IN: The Greenleaf Center.

Greenleaf, R. K. (1996). On becoming a servant-leader (D. M. Frick \& L. C. Spears, Eds.). San Francisco, CA, US: Jossey-Bass.

Hair, J. F., Hult, G. T. M., Ringle, C. M., \& Sarstedt, M. A. (2016). Primer on partial least squares structural equation modeling (PLS-Sem). Thousand Oaks, CA: Sage.

Harter, S. (2002). Authenticity. In C. R. Snyder \& S. J. Lopez (Eds.), Handbook of positive psychology (pp. 382-394). New York, NY: Oxford University Press.

Hayes, B. E. (1998). Measuring customer satisfaction: Survey design, use, and statistical analysis methods. Milwaukee, Wisconsin: ASQC Quality Press.

Hernandez, M. (2008). Promoting stewardship behavior in organizations: A leadership model. Journal of Business Ethics, 80(1), 121-128. doi: 10.1007Л10551-007-9440-2

Hu, L. \& Bentler, P. (1999). Cutoff criteria for fit indices in covariance structure analysis: conventional criteria versus new alternatives. Structural Equation Modeling, 6(1), 1-55. doi: 10.1080/10705519909540118

Jorgensen, T. D., Pornprasertmanit, S., Schoemann, A. M., \& Rosseel, Y. (2018). semTools: Useful tools for structural equation modeling. R package version 0.5-1. Recuperado de https://CRAN.R-project.org/package $=$ semTools

Liden, R. C., Wayne, S. J., Zhao, H., \& Henderson, D. (2008). Servant leadership: Development of a multidimensional measure and multilevel assessment. Leadership Quarterly, 19(2), 161-177. doi: 10.1016/j.leaqua.2008.01.006

Marôco, J., \& Garcia-Marques, T. (2006). Qual a fiabilidade do alfa de Cronbach? Questões antigas e soluções modernas? Laboratório de Psicologia, 4(1), 65-90. doi: 10.14417/lp.763

Meyer, J. P., \& Allen, N. J. (1991). A three-component conceptualization of organizational commitment. Human Resource Management Review, 1(1), 61-89. doi: 10.1016/1053-4822(91)90011-Z

Muthén, L K, \& Muthén, B. O. (2010). Mplus: Statistical analysis with latent variables. User's guide. Los Angeles, CA: Muthén \& Muthén.

Patterson, K. A. (2003). Servant leadership: A theoretical model (Doctoral Dissertation). Regent University, Vírginia (UMI No. 3082719. Recuperado de https://www.regent.edu/acad/global/publications/sl_proceedings/2003/patterson_servant_leadership.pdf

Peterson, C., \& Seligman, M. E. P. (2004). Character strengths and virtues. $\bar{A}$ handbook and classification. Oxford, PÄ: Oxford University Press.

Rodríguez-Carvajal, R., de Rivas, S., Herrero, M., Moreno-Jiménez, B., \& Van Dierendonck, D. (2014). Leading people positively: Crosscultural validation of the Servant Leadership Survey (SLS). The Spanish Journal of Psychology, 17, E63. doi: 10.1017/sjp.2014.73

Rosseel, Y. (2012). Lavaan: An R package for structural equation modeling. Journal of Statistical Software, 48(2), 1-36. Recuperado de http:// www.jstatsoft.org/v48/i02/

Schaufeli, W. B., \& Bakker, A. B. (2003). UWES - Utrecht Work Engagement Scale: Test manual. Utrecht University, Department of Psychology. Recuperado de http://www.wilmarschaufeli.nl

Schaufeli, W., Salanova, M., González-Romá, V., \& Bakker, A. B. (2002). The measurement of engagement and burnout: A two sample confirmatory factor analytic approach. Journal of Happiness Studies, 3(1), 71-92. doi: 10.1023/A:1015630930326

Silva, A. P. C., \& Ferreira, M. C. (2009). Escala de satisfação geral no trabalho [Resumo]. In Instituto Brasileiro de Avaliação Psicológica (Ed.), Anais do IV Congresso Brasileiro de Avaliação Psicológica (p. 246). Campinas, SP: IBAP.

Sobral, F. J., \& Gimba, R. F. (2012). As prioridades axiológicas do líder autêntico: Um estudo sobre valores e liderança. Revista de Administração Mackenzie, 13(3), 96-121. doi: 10.1590/S1678-69712012000300006

Sousa, M., \& Van Dierendonck, D. (2014). Servant leadership and engagement in a merge process under high uncertainty. Journal Organizational Change Manage. 27(6), 877-899. doi: 10.1108/JOCM-07-2013-0133

Sousa, M., \& Van Dierendonck, D. (2015). Introducing a short measure of shared servant leadership impacting team performance through team behavioral integration. Frontiers in Psychology, 6, 2002. doi: 10.3389/fpsyg.2015.02002

Streiner, D. I. (2003). Starting at the beginning: An introduction to coefficient alpha and internal consistency. Journal of Personality Assessment, 80(1), 99-103. doi: 10.1207/S15327752JPA8001_18

Van Dierendonck, D. (2011). Servant leadership: A review and synthesis. Journal of Management, 37(4), 1228-1261. doi: $10.1177 / 0149206310380462$

Van Dierendonck, D., \& Nuijten I (2011). The Servant Leadership Survey: Development and validation of a multidimensional measure. Journal of Business and Psychology, 26(3), 249-267. doi: 10.1007/s10869-010-9194-1

Van Dierendonck, D., Haynes, C., Borrill, C., \& Stride, C. (2004). Leadership behavior and subordinate well-being. Journal of Occupacional Health Psychology, 9(2), 165-175. doi: 10.1037/1076-8998.9.2.165

Van Horn, J. E., Taris, T. W., Schaufeli, W. B., \& Schreurs, P. J. G. (2004). The structure of occupational well-being: A study among Dutch teachers. Journal of Occupational and Organizational Psychology, 77(3), 365-375. doi:10.1348/0963179041752718

Walumbwa, F. O., Avolio, B. J., Gardner, W. L., Wernsing, T. S., \& Peterson, S. J, (2008). Authentic leadership: Development and validation of a theory-based measure. Journal of Management, 34(1), 89-126. doi: 10.1177/0149206307308913

\section{Sobre as autoras}

Michelle Morelo Pereira é psicóloga (UFMT), mestre e doutoranda em Psicologia (UNIVERSO). Áreas de atuação: psicometria, avaliação psicológica, liderança, psicologia positiva e comportamento organizacional positivo.

Maria Cristina Ferreira é psicóloga, doutora em Psicologia (FGV), professora e coordenadora do Programa de Pós-Graduação em Psicologia na UNIVERSO. Áreas de atuação: comportamento organizacional positivo e psicometria. 